\title{
Retraction
}

\section{Effect of dietary manganese on antioxidant status and expressions of heat shock proteins and factors in tissues of laying broiler breeders under normal and high environmental temperatures - RETRACTION}

\author{
Yong-Wen Zhu ${ }^{1,2}$, Lin $\mathrm{Lu}^{1}$, Wen-Xiang $\mathrm{Li}^{1}$, Li-Yang Zhang ${ }^{1}$, Cheng $\mathrm{Ji}^{2}$, Xi Lin ${ }^{3}$, Hsiao-Ching Liu ${ }^{3}$, \\ Jack Odle ${ }^{3}$ and Xu-Gang Luo ${ }^{1}$ \\ ${ }^{1}$ Mineral Nutrition Research Division, Institute of Animal Science, Chinese Academy of Agricultural Sciences, Beijing 100193, \\ People's Republic of China \\ ${ }^{2}$ College of Animal Science and Technology, China Agricultural University, Beijing 100193, People's Republic of China \\ ${ }^{3}$ Department of Animal Science, North Carolina State University, Raleigh, NC 27695, USA
}

(First published online 6 December 2017)

doi:10.1017/S0007114516003822, Published by Cambridge University Press, 28 November 2016

As a result of a technical error at the Publisher, the same article was erroneously published twice, once in, vol. 114, issue 12, pp. 1965-1974 and again in vol. 116, issue 11, pp. 1851-1860. Subsequently it is necessary to retract the second appearance of the article in favour of the first for there to be a single version of record, though there is no issue with the article itself. Cambridge University Press sincerely apologise to the authors and readers for this mistake.

\section{References}

1. Zhu Y., Lu L., Li W., Zhang L., Ji C., Lin X., ... Luo X. (2015) Effect of dietary manganese on antioxidant status and expression levels of heat-shock proteins and factors in tissues of laying broiler breeders under normal and high environmental temperatures. British Journal of Nutrition 114(12), 1965-1974. doi: 10.1017/S0007114515003803.

2. Zhu Y., Lu L., Li W., Zhang L., Ji C., Lin X., ... Luo X. (2016) Effect of dietary manganese on antioxidant status and expressions of heat shock proteins and factors in tissues of laying broiler breeders under normal and high environmental temperatures. British Journal of Nutrition 116(11), 1851-1860. doi: $10.1017 /$ S0007114516003822. 